\title{
Enhanced Passive RFID Based Disaster Management for Coal Miners
}

\author{
Amith S. Bhat, B. Raghavendra, and G. Narendra Kumar
}

\begin{abstract}
Solutions exist to cater the various aspects of an emergency response in coal mines that are subjected to the quality and availability of relevant information often oblivious due to inevitable and unpredictable nature of disasters. The major challenge is to implement a scheme which is economically viable, reasonably accurate and modestly enforced with least maintenance. Inexpensive Passive RFID (Radio Frequency Identification) tags offer a simplistic yet elegant approach in cumulating pertinent data which primarily aids in potentially disastrous situations and also serves to increase productivity in mundane activity of the mine. This paper presents a novel concept of organizing and interpreting the cognition of identities employing passive RFID tag with an additional radio frequency energy harvesting module capable of delivering power upto $-19 \mathrm{dBm}$ in turn fostering the range and readability figures. The first step towards a palpable database to make dynamic evacuation plan a substantial reality is emphasized. A comparative study is shown between a normal RFID tag and the enhanced one, which exhibits promising results.
\end{abstract}

Index Terms-Event, LIMD (location and identity mapping database), node, RF Harvesting, RFID (radio frequency identification), RFID reader, RFID Tag, sector

\section{INTRODUCTION}

In case of an underground mine disaster situation, it is a difficult task for the mine management to track and identify the personnel trapped without knowing their exact location which impels the proposition of a new approach. The mining industry is capital intensive, the cost of maintenance at mechanized mines being 50-60\% when both direct and indirect costs are taken into account [1]. The present day globally competitive market scenario encourages the mining industry in automation, optimum utilization of equipment and nominal use of man power [2].

Radio frequency identification is playing a major role in the contemporary consumer industry. While much work on RFID applications has been done worldwide, it is yet to make its mark in disaster management, especially in the mining sector. Constraints on economy, maintenance and lack of a reliable ideology involving the location indexing of personnel at the imminent moment of disaster has apparently decimated the chances of incorporation of RFID technology for such an application.

Gathering radio frequency electromagnetic radiation and using it either to directly power a circuit or storing it for

Manuscript received December 14, 2012; revised February 23, 2013.

The authors are with University Visvesvaraya College of Engineering, Bangalore University, Bangalore India (e-mail: sbamith1991@gmail.com, raghavendrabsrg@gmail.com, gnarenk@yahoo.com).z later use for low power electronic applications is a flourishing practice [3]. This elementary technique can benevolently be used to overcome the conventional drawbacks of passive RFID such as diminished range and low power availability in the passive RFID tag.

\section{RFID}

Radio Frequency Identification (RFID) is the usage of a wireless system that uses radio-frequency electromagnetic fields to transfer highly specific data from a tag attached to an object, for the purposes of automatic identification and tracking. The tag contains electronically stored information which can be read from up to several meters away. Unlike a bar code, the tag does not need to be within line of sight of the reader and may be embedded in the tracked object. RFIDs are broadly assorted into Active and Passive types. Essentially, active and passive RFID both use RF (Radio Frequency) energy for communication between a tag and a reader device. But the methods of powering the tags differ. Active RFID uses a battery within the tag; passive RFID depends on RF energy transmitted by the reader to power the tag. Although the active RFIDs are effective in long range reliable transmission, the requirement of an onboard power source which has to be periodically replenished confines its usage [4]. Passive RFIDs with no onboard power source are operative only in short range, making them not completely dependable. Incorporating the vantages of both types is the Semi-passive RFID which uses a battery to power onboard circuitry or sensors but not to generate back channel signals, i.e., the channel on which RFID tag transmits the signal, but the usage is hindered due to higher costs. Since tags have individual, unique serial numbers called the Electronic Product Code (EPC), the RFID system design can differentiate several tags that might be within the range of the RFID reader and read them rapidly.

\section{A. RFID Reader}

RFID Reader is a device which has one or more antennas that emit interrogator signals, receives the EPC back from the tag and passes the information in digital form to a computer system for processing. A standard commercially available reader $V F-647$ is considered with regard to its specifications as:

Tag air interfaces EPC global UHF Class 1 Gen 2 / ISO 18000-6C/ ISO18000-6B protocols; frequency ranges of 902-928MHz; RF power control of +10.0 to $+32.5 \mathrm{dBm}$; read distance of 3-20 meters; maximum receiving sensitivity of $-82 \mathrm{dBm}$; maximum return loss of $10 \mathrm{dBm}$; communication interfaces LAN, RS232, RS485; communication serial 
port rate of 9600-115200bps; works with external power of $22 \mathrm{OV}$ ac, $+12 \mathrm{~V} / 3 \mathrm{~A}$ dc adapter consuming average power less than $20 \mathrm{~W}$; operating temperature -20 to $+60{ }^{\circ} \mathrm{C}$; humidity $5 \%$ to $95 \%$; dimensions $220(\mathrm{~L}) \mathrm{mm} \quad \mathrm{x}$ $200(\mathrm{~W}) \mathrm{mm} \times 48(\mathrm{H}) \mathrm{mm}$; weighing $1.5 \mathrm{Kg}$. The ability to collect information from multiple tags, functionality in metallic, wet environments, and integration with other sensor technologies are crucial factors indeed.

\section{B. Passive RFID Tag}

Commercially available passive RFID tags are restricted in terms of range and power. Its indispensable advantage being that it is cost effective. In the ongoing discussions, a commercially available passive low power RFID $M R 6700 A / B$ is utilized with the following specifications:

Supports transponders of ISO18000-6B; ISO18000-6C EPC GEN2 protocols with a frequency range of ISM $860-960 \mathrm{MHz}$; with a memory capacity of 2048 bits with average reading rate of $64 \mathrm{bits}$ in $10 \mathrm{~ms}$; reading range being approximately $8 \mathrm{~m}$ at operation temperature -20 to $80^{\circ} \mathrm{C}$. Compact size and very low cost favor their usage in large scale mining operations. Enhancement of range and readability of the tag keeping other factors intact is primarily focused. A typical passive RFID internal hardware block diagram is explored, Fig. 1

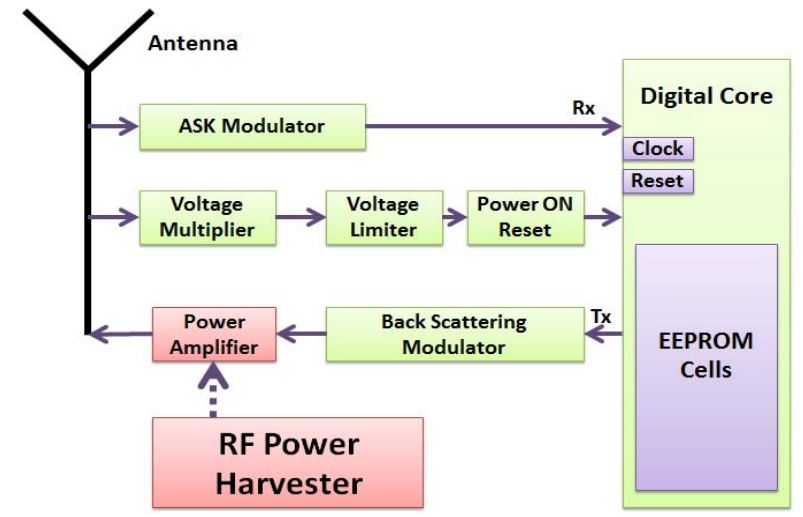

Fig. 1. RFID tag Internal block diagram with additional power harvester module.

An analog front end where the antenna is connected to an ASK demodulator receiving synchronizing signals from the reader is seen. A voltage multiplier circuit to enhance voltage level, a transmitter for back scattering transmission, a digital block storing the EPC in the EEPROM cells and certain control logic to regulate the operations is present. The dotted arrow indicates the usage of additional stage of power amplifier which draws its energy from an external power harvesting module and amplifies the backscattering signal [5].

\section{IMPLEMENTATION}

Following is an exploration of elements of the system implemented so as to leverage performance from the presently available elementary models.

\section{A. RF harvesting circuitry}

$\mathrm{RF}$ harvesting is the principle wherein the radio frequency electromagnetic radiation are effectively tapped and converted into electrical energy. The concept requires an efficient antenna along with a circuit capable of converting RF power into usable form.

1) Power harvesting antenna: In communication systems, antennas are purported to receive a specific band of frequencies which generally contain data. However the power harvesting application requires that all possible bands of frequencies are tapped into, including radio frequency noise. The implication of this means that effective power is an additive function of respective powers received. A log-periodic spiral antenna, Fig.2, is chosen due to the reasons below [6].

- Broadband antenna.

- Uni-planar, convenient for circuit connection.

- Omni-Directional radiation pattern.

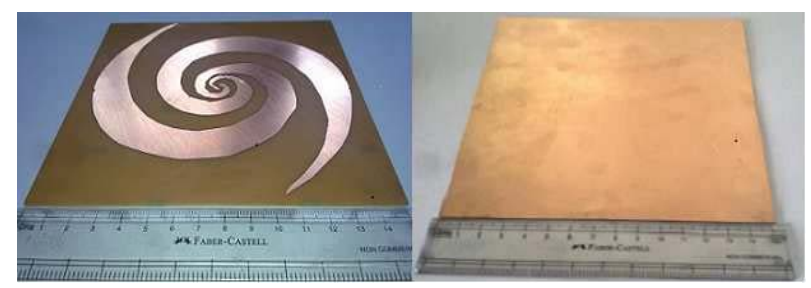

Fig. 2. Spiral antenna constructed on PCB with its ground plane

Design equations:

$$
\begin{aligned}
f_{l} & =\frac{c}{2 \Pi R_{o}} \\
f_{h} & =\frac{c}{4 R_{i}}
\end{aligned}
$$

where ' $c$ ' is the velocity of light, $\mathbf{f}_{\mathbf{l}}$ and $\mathbf{f}_{\mathbf{h}}$ are the lowest and highest harvestable frequency, $R_{o}$ and $R_{i}$ are the outer and inner radii of the spiral respectively.

By considering $f_{1}$ as $600 \mathrm{MHz}$ and $f_{h}$ as $10 \mathrm{GHz}$, $R_{\mathrm{o}}$ as $7.5 \mathrm{~cm}$ and $R_{i}$ as $0.75 \mathrm{~cm}$ is obtained.

An effective antenna design includes a mounted ground plane which is a conductive surface usually connected to electrical circuit ground. The planar spiral antenna considered is implemented on a double sided printed circuit board. The antenna resides on one of the surfaces while the other surface is made to act as the ground plane. Under normal circumstances, a circuit is to be contrived such that the antennas impedance matches with that of the load, failing which there will be reflection of power back into the antenna. For this purpose, matching networks are used to ensure maximum power transfer to the load. The matching network in this case is obsolete, since the log-periodic spiral antenna designed above gives almost constant impedance for the chosen frequency range [7].

2) Voltage doubler and charging circuit: A voltage doubler is used to convert the induced RF power into dc power and a series of doublers are subsequently used to increase the voltage levels so as to meet the required voltage specifications of a standard rechargeable $\mathrm{Ni}-\mathrm{MH}$ (Nickel-Metal Hydride) button cell with cell voltage $1.2 \mathrm{~V}$ and capacity of $100 \mathrm{mAh}$. During the experiment, a voltage of $50 \mathrm{mV}$ was obtained from the spiral antenna without any 
voltage doubler, measured at 10 meter distance from a $V F-647$ RFID reader. A total of 5 stage voltage doublers were employed resulting in $1.6 \mathrm{~V}$ which is sufficient to start charging the button cell.

The estimated incident power on the physical antenna aperture was found to be around $0.12 \mu \mathrm{W} / \mathrm{cm}^{2}$ whose effective aperture being around $100 \mathrm{~cm}^{2}$ resulting in $12 \mu \mathrm{W}$ $(-19 \mathrm{dBm})$ of power

Being fed to the button cell The above reading was obtained in an environment consisting of frequencies characteristic to the reader, i.e., $902-928 \mathrm{MHz}$, as well as external broadcast frequencies. In the absence of any electromagnetic interference, an output of $38 \mathrm{mV}$ was obtained solely due to the RFID reader.

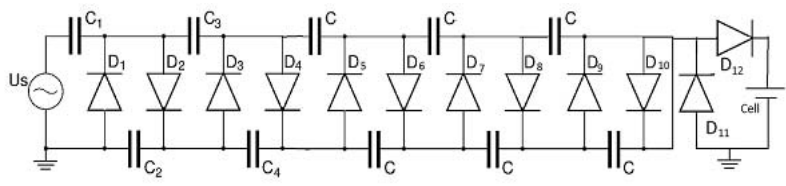

Fig. 3. 5 Stage voltage doubler and battery charging circuit

The circuit depicted above uses 10 electrolytic capacitors of $1 \mu \mathrm{F}$ and $25 \mathrm{~V}, 11$ low voltage drop 0A79 Germanium diodes(D1-D10) for 5 stage voltage doubling, (D11) is to prevent battery discharge in the circuit path, (D12) is a zener diode with 1.2 volts reverse voltage level to keep the voltage across it a constant.

\section{B. LIMD [Location and Identity Mapping Database]}

A readily available index of information is of utmost requirement for conducting emergency rescue procedures during the initial phase of the rescue [8], [9].

LIMD is defined as an event driven database of Location and Identity representation in an easy readable format. The database is created by implementing a system of RFID tag readers, as depicted

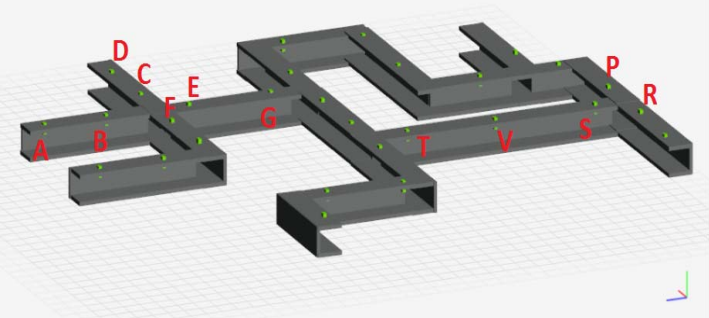

Fig. 4. 3D terrain representation with readers labeled

The green blocks symbolize the RFID readers placed strategically at certain distance within the tunnel, with each reader being declared as a unique Node and the tunnel space between two nodes is a unique Sector. The reading range of each individual reader must be non-overlapping. In case of multiple tunnels emerging from a junction, it is positioned at a certain distance from the junction to abide the above assertion. An Event is defined to occur, if the EPC of an RFID tag, either of personnel or machinery is read by a node. A set of inexpensive sensors, for instance Infrared detectors could be placed on either ends of the RFID reader's range extremities to detect any motion. In the case of motion being detected, the RFID readers are designed to automatically switch on. The RF power is synthesized in the harvesting circuit placed on the helmet of the miner as a result of which the tag is read with a heightened readability and improved range factor. The infrared sensors ensure that the readers are idle and power is saved when no person or machinery is within the reading range. The reader is programmed to have an optimum latency period during which it scans for any RFID tags within its range, after which it shuts itself down.

An illustration is shown regarding the event driven operation and subsequent table updates of LIMD, Fig. 5.

\begin{tabular}{|c|c|c|c|c|c|}
\hline Tag ID & PID & PN & $\mathrm{CN}$ & NPN & Location ID \\
\hline 100001 & $\begin{array}{l}\text { WD_15 } \\
\end{array}$ & $\overline{A A}$ & $\bar{B}$ & 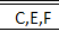 & 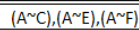 \\
\hline 100002 & ENI_23 & $\mathrm{T}$ & $\mathrm{v}$ & $\mathrm{s}$ & $\left(T^{\sim} \mathrm{S}\right)$ \\
\hline Tag ID & PID & PN & $\mathrm{CN}$ & NPN & Location ID \\
\hline 100001 & WD_15 & B & $\bar{E}$ & $\mathrm{C}, \mathrm{F}, \mathrm{G}$ & $\left(\mathrm{B}^{\sim} \mathrm{C}\right),\left(\mathrm{B}^{\sim} \mathrm{F}\right),\left(\mathrm{B}^{\sim} \mathrm{G}\right)$ \\
\hline 100002 & ENI_23 & v & $\mathrm{s}$ & $P, R$ & $\left(\mathrm{~V}^{\sim} \mathrm{P}\right),\left(\mathrm{V}^{\sim} \mathrm{R}\right)$ \\
\hline Tag ID & PID & PN & $\mathrm{CN}$ & NPN & Location ID \\
\hline 100001 & $\begin{array}{l}\text { WD_15 } \\
\end{array}$ & $\bar{E} \mathrm{E}$ & $\bar{c} \mathrm{C}$ & $\mathrm{B}, \mathrm{D}, \mathrm{F}$ & 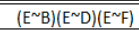 \\
\hline 100002 & ENI_23 & $\mathrm{s}$ & $\mathrm{v}$ & $\mathrm{T}$ & $\left(S^{\sim} T\right)$ \\
\hline
\end{tabular}

Fig. 5. LIMD Table updating process

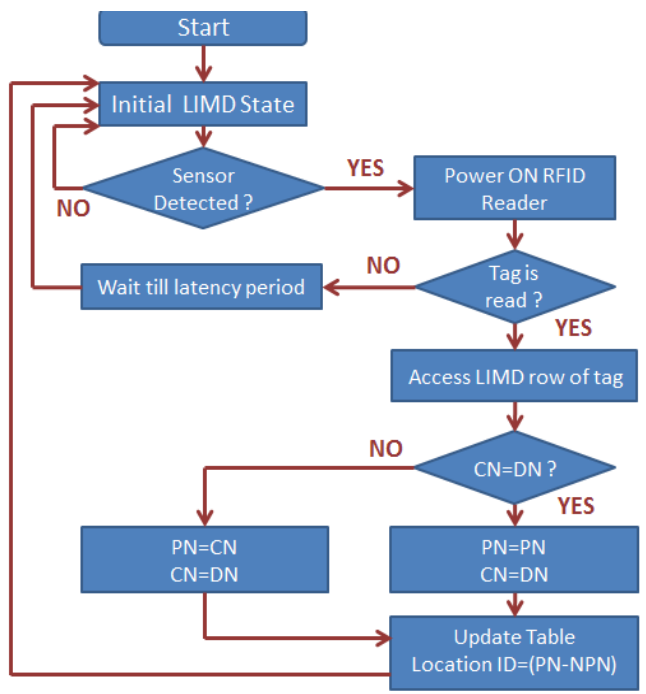

Fig. 6. The LIMD flowchart

where $C N$ is current node, $P N$ is past node, $N P N$ is next possible node and $P I D$ is an assigned personnel ID of the miner.

The first column represents the unique RFID tags distributed to concerned individuals, each represented by their assigned personnel ID in the second column. The next two columns indicate the nodes traversed in succession. The fifth column indicates possible nodes which can be encountered in the geography considering the previous and current nodes.

The definitive order of arrangement of the nodes is pre-known to the database software through the initial geographical survey. A scenario is considered as seen in the table above also depicted diagrammatically, Fig.4. The personnel referred by the Tag ID 100001 initially is at node $\mathrm{B}$ having passed node $\mathrm{A}$. The next possible nodes could be C, E, or F. With the knowledge of all the nodal data from the columns 3,4 , and 5 , the possible sectors between the nodes where the miner is occupied can be approximated as any among (B-C), (B-E), or (B-F). Since there is also a possibility of retracing the previous node, the miner can be in either (A-B), (B-C), (B-E), or (B-F). Therefore, taking this 
into account, the effective location ID can be judged as (A-C), (A-E), or (A-F).

The respective rows of consecutive tables are then updated individually for every trigger of an event due to which the current node value is updated to previous node value. The detected node (DN) assumes the current node in the table and the location ID is updated depending on the next possible nodes as visualized in the flow chart.

A base station located on the surface is hardwired to all the RFID readers which serve to power the readers. The processing of information channeled through an adjacent wired medium is done by the server at the base station, Fig. 7.

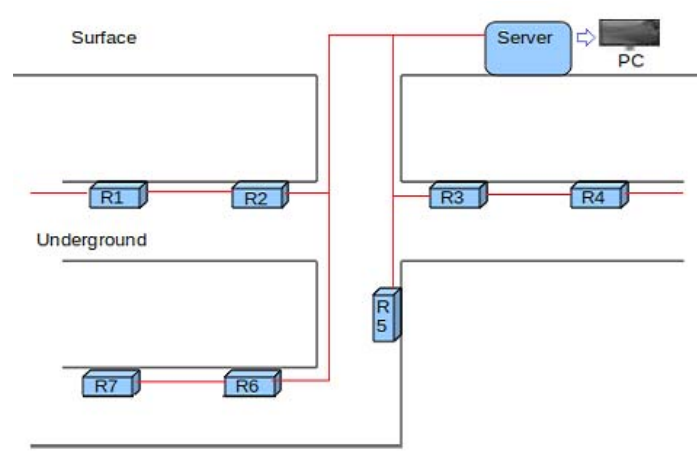

Fig. 7. Linking the readers to server

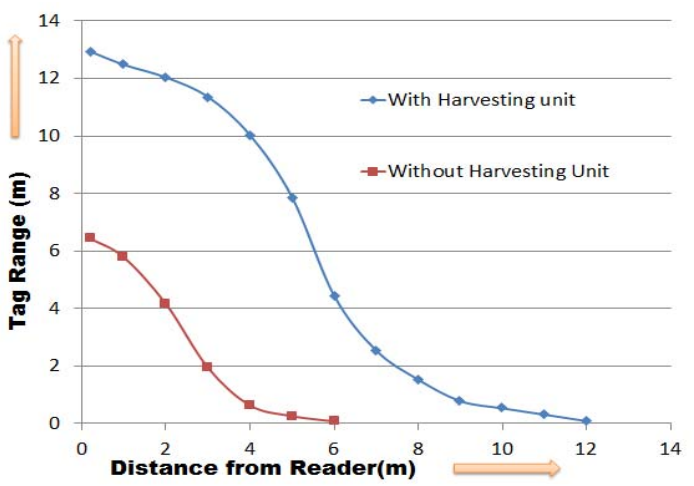

Fig. 8. A graph depicting experimental result of tag range as a function of its distance from the reader

Software which maintains and updates the database in alliance with received data from all the readers is incorporated in the server. This software is equipped with a visually mapped geographical data for reference. Periodic refreshing of acquired data is needless since updating of the database is event driven. With the site of disaster known, relevant information concerning number of trapped victims and their identification can be easily extracted using this database, facilitating a speedy and smart rescue operation.

\section{Simulation AND Results}

\section{A. Range Comparison with and without Power Harvester}

A comparative study is presented after conducting experimentation on the actual hardware considering cases of a traditional passive RFID tag and an enhanced one. A plot of the tag range as a function of distance from reader for the above two cases is plotted, Fig.8.
As one can observe, the effective tag range for an enhanced passive RFID far exceeds that of a traditional one when measured at the same distance from the reader under similar conditions. The power available at the enhanced passive RFID tag transmitter for backscattering transmission is measured to be $0.0008 \mu \mathrm{W} / \mathrm{cm}^{2}\left(-61 \mathrm{dBm} / \mathrm{cm}^{2}\right)$ at a distance of $10 \mathrm{~m}$ from the tag as compared to $0.00009 \mu \mathrm{W} / \mathrm{cm}^{2}$ of a traditional passive RFID tag, increasing the range by threefold on an average. The RFID reader is capable of receiving backscatter signals of upto -82 $\mathrm{dBm}$ therefore the enhanced passive RFID has an elevated chance of detection. Since the available power to the tag transmitter is more, the Signal-to-Noise Ratio (SNR) associated with transmission is more, this enhances the readability and the back scattering transmission is less prone to errors.

\section{B. LIMD Case Study}

For the scenario presented in Fig.5, a simple simulation is performed using the software Network Simulator 2. When the miner is not in range of node $\mathrm{B}$, communication is absent and the LIMD table remains unaltered, Fig.9. As the miner is approaching the node $\mathrm{B}$, the reader interacts with the RFID tag initiating the event, thus updating the LIMD table, Fig. 10.

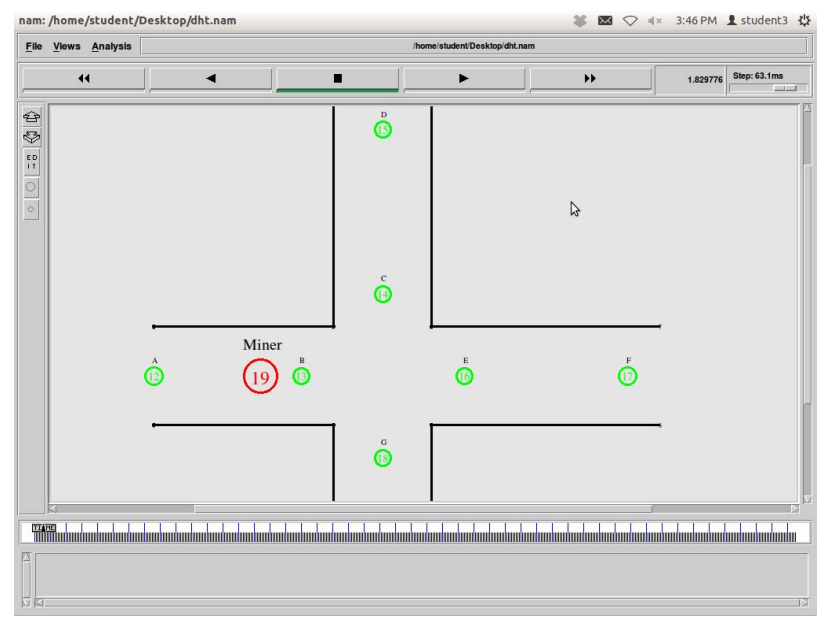

Fig. 9. NS2 screenshot indicating no event

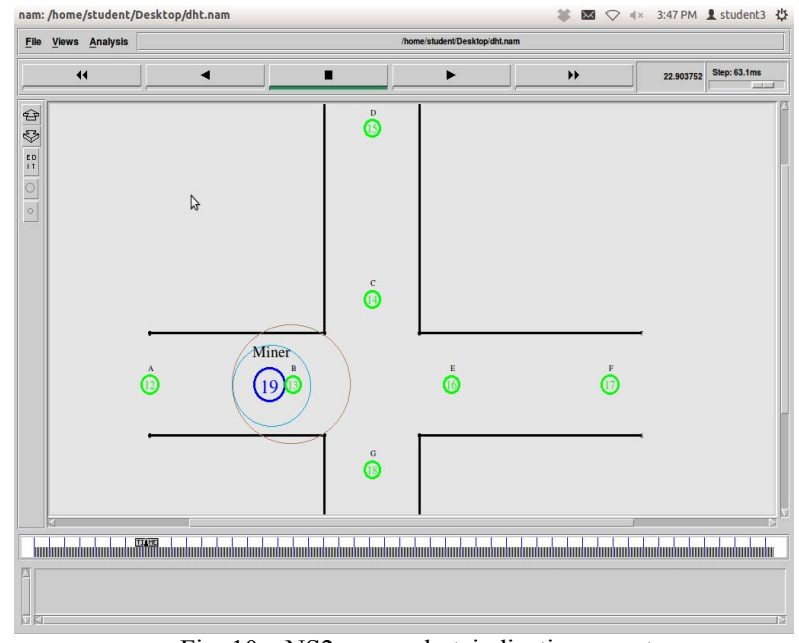

Fig. 10. NS2 screenshot indicating event

\section{CONCLUSION AND FUTURE WORK}

The credibility of using a power harvesting module in the 
system is to exploit the energy sources to the fullest and expend thus obtained energy for augmenting the readability of the RFID tag while maintaining complete mobility. The analogy of using LIMD database is to have a standard reference with applicable data to point out the intricate location. This paper has showcased the significance of using passive RFID tag for coal mine disaster situations and its ability to blend with additional components, boosting the overall performance parameters. The highlights of the proposed system comprehend cost effectiveness, energy efficiency of tag and reader alike, range and power maximization at the cost of least additional expenses and a readily available simplistic, perceptible database. In addition, it also serves the mine management to analyze the production pattern and initiate the concerned improvements.

The performance attributes of the system implementation can be immensely enriched, exploring several possible factors like better user interface in a graphical display form of the map in lieu to tabular form.

A promising feature to be probed into could be the establishment of suitable networking between the tags. The usable form of energy garnered until the impending moment of disaster which may be endowed for a short term adroit is thought evoking.

\section{REFRENCES}

[1] L. K. Bandyopadhyay, S. K. Chaulya, and P. K. Mishra, "Wireless informa tion and safety system for mines," Journal of Scientific \& Industrial Research," pp. 109-110, November 2008.

[2] G. NarendraKumar, G. K. Rama Murali, D. V. Sriharsha, and S. Srinivas, "An Efficient Disaster Management System for Coal miners using Sensor MANETs," presented at the Fifth International Conference on Industrial and Information Systems, NITK, Suratkal, India, 29th July-01st August 2010.

[3] B. R. Shashank, G. S. Kumar, K. Kalaiarasi, A. Shalini, and A. R. Prabhu, "Implementation of Ambient Energy Harvesting from Air for ULP Devices as a Renewable Energy source," presented at the 2nd
International conference on Environmental science and development, 2011.

[4] F. Feng, H. Shengyu, and X. Qi, "The Research of the ZigBee and RFIDFusion Technology in the Coal Mine Safety," International Conference on Innovation and Management, 2010.

[5] V. Chawla and D. S. Ha, "An Overview of Passive RFID," Communications Magazine IEEE, pp. 11-13, 2007.

[6] D. Bouchouicha, F. Dupont, and M. Latrach, "Ambient RF Energy Har vesting," in Proc. International Conf. on Renewable Energies and power quality, Bucharest Bucharest, Romania, pp. 2-5, 2011.

[7] T. Paing, J. Shin, R. Zane, and Z. Popovic, "Resistor Emulation Approach to Low-power RF Energy Harvesting," IEEE trans- actions on power electronics, pp. 3-5, 2008.

[8] W. Burgard, D. Fox, K. Fishkin, and M. Philipose, "Mapping and localization with RFID technology," in Proc. Robotics and Automation, ICRA, pp. 3-7, 2004

[9] Z. Qinghua, W. Bo, C. Guoquan, and W. Zhuan, "Object position tracking based on e-map and RFID in coal mine," presented at ICIEA, 2009.

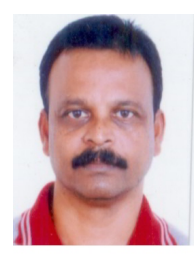

Narendra Kumar G. is a professor in Bangalore University, Bangalore, India. He completed his Master of Engineering Degree in Electrical Communication Engineering, (Computer Science \& Communication) in 1987 at IISc, Bangalore, India. Later in 2006 he obtained PhD in Electrical Engineering (Computer Networks) in 2006 in Bangalore University, Bangalore. His fields of specialization include Wireless Networks, Multimedia System, Mobile Communication, and Disaster Management.

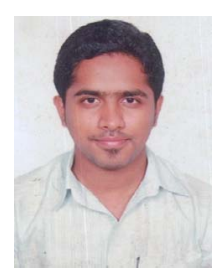

Amith S. Bhat was born on $25^{\text {th }}$ Oct 1991 in Bangalore, India. He is an undergraduate student of Electronics \& communications engineering in Bangalore University, Bangalore, India.

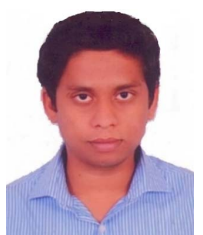

Raghavendra B. was born on $30^{\text {th }}$ Dec 1991 in Bellary, India. $\mathrm{He}$ is an undergraduate student of Electronics \& communications engineering in Bangalore University, Bangalore, India. 